\title{
Immortality and other stories: the dual role of telomerase
}

Cancer cells must achieve immortality in order to propagate, and this generally occurs by reactivating telomerase - an enzyme that comprises two subunits, mTert and mTerc, and that prevents replicative senescence and crisis (two responses that occur as telomere reserve is exhausted) by maintaining telomere length. But the telomerase story might not be quite as simple as was first thought; Steven Artandi et al. have recently reported that telomerase activity can promote tumorigenesis independently of telomere length.

Transgenic mice were generated that express the catalytic mTert subunit, and the mRNA was shown to be upregulated - compared with that in wild-type mice - in several tissues, including thymus, lung, kidney, mammary glands, heart, muscle and brain . However, heart, muscle and brain tissues did not have a corresponding increase in telomerase activity - as determined by the telomerase repeat amplification protocol (TRAP) assay. One explanation for this could be the low expression levels of the mTerc subunit that were found in these tissues.

So what are the physiological effects of forced mTert expression, and hence telomerase activity? Mouse embryonic fibroblasts (MEFs) from either wildtype or $m$ Tert-expressing mice were used to show that the mean telomere length increased from $50 \mathrm{~kb}$ to $70 \mathrm{~kb}$ when $m$ Tert was expressed. But, interestingly, these cells undergo cultureinduced senescence - a growth arrest that occurs after several passages, and is thought to be independent of telomere length - at the same time and with the same kinetics as wild-type cells. This provides further support that culture-induced senescence, unlike replicative senescence in human cells, is not related to telomere length.

To determine how enforced telomerase expression affected tumorigenesis, wild-type and mTert transgenic mice, both of which had ample telomeres, were treated with the carcinogen dimethylbenz $[a]$ anthracene (DMBA). The high tumour incidence in wild-type mice was not further increased by the expression of $m$ Tert. Crossing mTert-transgenic mice with tumour-prone $C d k n 2 a^{-/-}$mice which are deleted for the Ink4a and Arf tumour suppressors - to generate $m$ Tert transgenic $C d k n 2 a^{-/}$mice neither increased tumour incidence nor altered the tumour spectrum from the sarcomas and lymphomas that are normally found in $C d k n 2 a^{--}$mice.

But could a subtle defect that occurs with mTert expression be masked in these highly cancer-prone mice? When monitored over a long period of time, the $m$ Tert-expressing mice were found to develop a significant number of breast carcinomas, as well as the normal spectrum of tumours that the wild-type mice develop when they reach old age. This is consistent with the increased telomerase activity in the breast tissue of the transgenic mice. Histological analysis revealed that $67 \%$ of one transgenic mouse strain and $33 \%$ of another, which expressed lower levels of $m$ Tert, developed mammary intraepithelial neoplasia (MIN) - a precursor to breast carcinoma - throughout the mammary gland, compared with none of the wild-type control mice.

Telomerase might therefore have a dual function in cancer cells: to lengthen short telomeres, allowing unlimited proliferation, and to promote tumorigenesis by an unknown, telomere-length-independent mechanism. Why this function seems to be restricted to mammary-gland cells, when telomerase activity is high in other tissues, is still to be determined; as is the pathway by which telomerase exerts this effect. This work can only encourage the development of telomerase inhibitors for cancer therapy.

$$
\text { Emma Greenwood }
$$

\section{(2) References and links} ORIGINAL RESEARCH PAPER Artandi, S. E. et al. Constitutive telomerase expression promotes mammary carcinomas in aging mice. Proc. Natl Acad. Sci. USA 99, 8191-8196 (2002) WEB SITES

Ron DePinho's lab:

http://www.hms.harvard.edu/dms/bbs/fac/ depinho.html

\section{TUMOUR SUPPRESSORS}

\section{Biallelic inactivation of BRCA2 in Fanconi anemia.}

Howlett, N. G. et al. Science 13 June 2002 [epub ahead of print]

The cancer-predisposition syndrome Fanconi anaemia (FA) can be caused by germ-line mutations in several genes. However, the genes that cause FA subtypes B and D1 have not been identified. Now, Alan D'Andrea and colleagues show that cell lines that are derived from patients with FA-B and FA-D1 have biallelic mutations in $B R C A 2$, which result in the expression of truncated proteins. This study adds $B R C A 2$ to the common pathway that has already been established for $B R C A 1$ and the six identified $F A$ genes.

\section{MELANOMA}

Bcl2 regulation by the melanocyte master regulator Mitf modulates lineage survival and melanoma cell viability.

McGill, G. G. et al. Cell 109, 707-718 (2002)

The SCF ligand, its receptor Kit, and the downstream transcription factor Mitf are important for the survival of melanocytes - a pathway that might be involved in the resistance to treatment that occurs in melanomas. A microarray-based screen used to identify Mitf transcriptional targets has led to the discovery that the antiapoptotic gene $B c l 2$ is directly regulated by Mitf; $B c l 2$ expression is also essential for melanoma survival. Mitf, in addition to Bcl2, could therefore be a useful theraputic target for melanoma.

\section{TUMOUR TARGETING}

A tumor-homing peptide with a targeting specificity for lymphatic vessels.

Laakkonen, P. et al. Nature Med. 7, 751-755 (2002)

This study describes a combined ex vivo and in vivo phage selection for peptides that recognize tumour vessels other than blood vessels. Using this screen, the authors isolated a cyclic 9-amino-acid peptide, LyP1, that specifically accumulates in the nuclei of tumour lymphatic cells in mice. LyP1 does not bind all tumour lymphatics, however, indicating that different tumours might have different types of lymphatic vessels. Lymphatic vessels are important routes for tumour metastasis, so LyP1 can be developed as a vehicle for delivering cytotoxic agents that block tumour expansion.

\section{ANGIOGENESIS}

Active immunization against the vascular endothelial growth factor receptor Flk1 inhibits tumor angiogenesis and metastasis.

Li, Y. et al. J. Exp. Med. 195, 1575-1584 (2002)

The vascular endothelial growth factor receptor-2 (VEGFR2/ FLK1) has a crucial role in tumour angiogenesis. Li et al. have developed an immunotherapy approach, using dendritic cells pulsed with soluble VEGFR2, to directly target the blood vessels around the growing tumour cells, which would cut off their oxygen supply. Inhibition of VEGFR2 suppressed tumour angiogenesis and development of metastases, and increased survival in mouse models. 